\title{
Carcinogen-induced hepatic tumors in KLF6+/- mice recapitulate aggressive human hepatocellular carcinoma, associated with p53 pathway deregulation
}

\author{
Mirko Tarocchi ${ }^{1,2}$, Rebekka Hannivoort ${ }^{1,3}$, Yujin Hoshida ${ }^{4}$, Ursula E. Lee ${ }^{1}$, Diana Vetter ${ }^{1}$, \\ Goutham Narla ${ }^{5,6}$, Augusto Villanueva ${ }^{7}$, Moshe Oren ${ }^{8}$, Josep M. Llovet ${ }^{1,7}$, and Scott L. \\ Friedman $^{1}$
}

${ }^{1}$ Department of Medicine/Division of Liver Diseases, Mount Sinai School of Medicine, New York, NY ${ }^{2}$ Department of Clinical Pathophysiology/Gastroenterology Unit, University of Florence, Florence, Italy ${ }^{3}$ Department of Gastroenterology and Hepatology, University of Groningen, Groningen, the Netherlands ${ }^{4}$ Cancer Program, Broad Institute of Massachusetts Institute of Technology and Harvard University, Cambridge MA ${ }^{5}$ Department of Medicine/Division of Hematology/Oncology, Mount Sinai School of Medicine, New York, NY ${ }^{6}$ Department of Genetics and Genomic Sciences, Mount Sinai School of Medicine, New York, NY ${ }^{7}$ HCC Translational Research Laboratory, Barcelona-Clinic Liver Cancer Group, Liver Unit. Institut d'Investigacions Biomediques August Pi i Sunyer (IDIBAPS); Centro de Investigación Biomédica en Red de Enfermedades Hepáticas y Digestivas (CIBEREHD), Hospital Clinic, Barcelona, Spain; Institució Catalana de Recerca i Estudis Avançats ${ }^{8}$ Department of Molecular Cell Biology, The Weizmann Institute, Rehovot, Israel

\section{Abstract}

Inactivation of KLF6 is common in hepatocellular carcinoma (HCC) associated with hepatitis C virus $(\mathrm{HCV})$ infection, thereby abrogating its normal anti-proliferative activity in liver cells. The aim of the study was to evaluate the impact of KLF6 depletion on human HCC and experimental hepatocarcinogenesis in vivo. In patients with surgically resected HCC, reduced tumor expression of KLF6 was associated with decreased survival. Consistent with its role as a tumor suppressor, KLF6 +/- mice developed significantly more tumors in response to the chemical carcinogen DEN than wild type animals. Gene expression signatures in both surrounding tissue and tumors of KLF6+/- mice closely recapitulated those associated with aggressive human HCCs. Expression microarray profiling also revealed an increase in mdm 2 mRNA in tumors from KLF6 +/compared to KLF6 +/+ mice, which was validated by real-time qPCR and Western blot in both human HCC and DEN-induced murine tumors. Moreover, chromatin immunoprecipitation and cotransfection assays established the $\mathrm{P} 2$ intronic promoter of $m d m 2$ as a bona fide transcriptional target repressed by KLF6. Whereas KLF6 over-expression in HCC cell lines and primary hepatocytes led to reduced MDM2 levels and increased p53 protein and transcriptional activity, reduction in KLF6 by siRNA led to increased MDM2 and reduced p53.

Conclusions-Our findings indicate that KLF6 deficiency contributes significantly to the carcinogenic milieu in human and murine $\mathrm{HCC}$, and uncover a novel tumor suppressor activity of KLF6 in HCC, by linking its transcriptional repression of MDM2 to stabilizing p53.

Corresponding Author: Scott L. Friedman, M.D., Division of Liver Diseases, Box 1123, Mount Sinai School of Medicine, 1425 Madison Ave., Room 11-70C, New York, NY 10029-6574; Fax 212-849-2574; Scott.Friedman@ mssm.edu.

Disclosures: The authors have nothing to disclose. 


\section{Keywords}

hepatic carcinogenesis; MDM2/HDM2; transcription factor; tumor biology

\section{Introduction}

Hepatocellular carcinoma (HCC) has a poor prognosis and is the third leading cause of cancer mortality worldwide. Development of pre-neoplastic lesions and their progression to HCC in patients with chronic liver disease reflect the convergence of genetic and epigenetic defects that provoke dysregulation of pathways controlling cell cycle, tissue repair and regeneration. Loss-of-heterozygosity (LOH) of tumor suppressor genes occurs commonly in HCC but no single tumor suppressor inactivation predominates. For example, LOH of p53 has been reported in only $\sim 25 \%$ of HCCs. For virtually all tumor suppressors, the presence of haploinsufficiency leads to an increased frequency of tumors in experimental models (1).

Inactivation of the KLF6 tumor suppressor has been implicated in several human cancers, including HCC (2-5). KLF6 is a member of the Krüppel-like C2H2 zinc finger (KLF) family, which regulate cell cycle, signal transduction and differentiation. KLFs, in particular KLF6, can serve as either trans-activators or trans-repressors depending on the cellular or developmental context (6). KLF6 mediates growth suppression through p53-independent p21 transactivation (2), sequestration of cyclin D1(7) and inhibition of the c-jun protooncogene (8).

HCC can harbor a range of genomic alterations and somatic mutations. In a minority of HCCs this includes structural defects in the p53 tumor suppressor (9), a nuclear phosphoprotein that regulates proliferation, maintenance of genomic stability, differentiation, apoptosis and microRNA processing. However, other pathways affecting p53 activity may be implicated. A major pathway regulating p53 homeostasis is its interaction with the E3 ubiquitin ligase MDM2/HDM2 (herein referred to by the mouse homologue, 'MDM2'). MDM2 directly binds p53, blocking the p53 transactivation domain and promoting its degradation (10). Conversely, p53 enhances $m d m 2$ transcription through its interaction with a pair of tandem p53-binding sites in $\mathrm{P} 2$ intronic promoter of the $m d m 2$ gene (11). These interactions comprise the auto-regulatory feedback loop controlling the steady state level and transcriptional function of p53 protein and the subsequent expression of the $m d m 2$ gene (12); the amplification or enhanced translation of $m d m 2$ confers transforming activity by inappropriately hastening p53 degradation. Indeed, the MDM2 gene is an oncogene frequently amplified and over-expressed in human tumors (13).

In characterizing the potential role of KLF6 in human HCC, our studies in a mouse model of the disease uncovered an inverse correlation between KLF6 and mdm2 mRNA expression, leading us to examine whether a functional interaction exists between these two proteins. Moreover, whereas previous studies indicated that KLF6 tumor suppressor activities are p53-independent (2), these new findings now directly link these two tumor suppressor pathways by demonstrating that KLF6 normally represses $m d m 2$ transcription, thereby stabilizing $\mathrm{p} 53$.

\section{Methods}

\section{Correlation of KLF6 mRNA expression with survival and recurrence of human HCC}

We analyzed KLF6 mRNA expression in $149 \mathrm{HCV}$-infected human samples representing the full pathological spectrum from normal to advanced HCC based on previously 
established criteria: human samples were obtained with full IRB consent as previously detailed (14-15).

We next examined the impact of KLF6 mRNA expression levels on survival and recurrence in 56 patients with $\mathrm{HCV}$-associated HCC by distinguishing between those tumors with 'very low' expression (expression < $10 \%$ that of normal livers) and 'low expression' (>10\% but $<50 \%$ that of normal livers). We chose specific, stringent mRNA cutoffs based on a similar approach in an earlier study (16) for two reasons: 1) to be sufficiently low to ensure biological relevance, since KLF6 expression in human HCC is extremely low and, 2) to preserve a sufficient number of patients in each group to allow meaningful comparisons ( $\mathrm{n}=16, \sim 30 \%$ of the total 56 patients analyzed for clinical correlations). Clinical characteristics of patients included in the outcome analysis are displayed in Supplemental Table 1.

\section{DEN model of experimental hepatocarcinogenesis}

Male KLF6 +/- mice (17) were bred with wild type C57BL/6 to generate mixed litters of KLF6 +/- and KLF6 +/+ animals. At 2 weeks of age, mice were injected intra-peritoneally with either a single dose of diethyl nitrosamine (DEN), $5 \mu \mathrm{g} / \mathrm{g}$ body weight in $100 \mu \mathrm{l}$ of saline, or vehicle alone. Mice were maintained on standard chow, and then sacrificed following ip injection of Avertin $(250 \mathrm{mg} / \mathrm{kg}) 3,6$ or 9 months later. At the time of sacrifice the animals were weighed, and blood and liver samples were harvested for analysis.

\section{Tumor quantification}

Macroscopic lesions were measured on the surface of the liver with a caliper at the time of sacrifice; the number, and size of tumors were then quantified. Hepatic tissues were fixed in $10 \%$ paraformaldehyde and embedded in paraffin: liver sections of $5 \mu \mathrm{m}$ were obtained from 4 different regions of the left lobe (at least $2 \mathrm{~mm}$ apart), stained with H\&E, and the surface area was quantified (BIOQUANT NOVA PRIME Measurement Software).

\section{Quantitative real-time PCR (RT-qPCR)}

Total RNA was extracted using Trizol reagent ${ }^{\mathrm{TM}}$ (Invitrogen, CA), followed by column purification (RNeasy ${ }^{\mathrm{TM}}$ mini kit, Qiagen, MD) and treated with DNAse (Roche, CT). A total of $0.5 \mu \mathrm{g}$ of RNA was reverse transcribed per reaction using Sprint ${ }^{\mathrm{TM}}$ RT Complete (Clontech Laboraties, CA). RT-qPCR was performed on ABI PRISM 7900HT Sequence Detection System (Applied Biosystems, CA). Primers sequence are listed in Supplemental Methods.

\section{Western blot}

Liver fragments were lysed in Lysis M buffer (Roche), then sonicated and pelleted. Supernatants were denatured; $40 \mu \mathrm{g}$ of protein was separated by polyacrylamide gel electrophoresis, and then transferred to nitrocellulose membranes (Invitrogen). Membrane blotting was performed using rabbit polyclonal antibody to KLF6 and GAPDH (Santa Cruz Biotechnology, CA), Calnexin (Abcam, MA) or mouse monoclonal antibody to MDM2 and p53 (Calbiochem, Germany) and $\beta$-tubulin (Sigma-Aldrich, MI).

\section{Chromatin immunoprecipitation assay}

HepG2 chromatin complexes were co-immunoprecipitated with KLF6 or IgG control antibodies. The DNA resulting from the precipitation was detected using primers surrounding the putative KLF6-binding site (MDM2-P2) or another upstream region (MDM2-P1) of MDM2 promoter (primers sequence available in Supplemental Methods). 


\section{Cell culture and transfection}

HepG2, Huh7 and Hep3B were cultured in standard conditions. Primary hepatocytes were isolated, cultured and transfected as indicated in Supplemental Methods. Transient transfection of cell lines was performed with Lipofectamine 2000 reagent (Invitrogen) according to the manufacturer's instructions. Cells were transfected with pCIneo vector, pCIneo-KLF6 vector (containing the full length human KLF6 cDNA), pSuper-siControl, pSuper-siKLF6 (18), pSN3-p53, different $m d m 2$ promoter luciferase reporters (P1, P2, $\mathrm{P} 1+\mathrm{P} 2)(19)$, or P2-mut $m d m 2$ promoter luciferase reporter (in which two KLF6 binding sites were mutated from CACCC to CACTC using Stratagene Quikchange XL) or Cyclin G1luciferase promoter (20). Renilla luciferase vector (Promega, WI) was used as internal control.

\section{Microarray analysis}

Gene expression analysis was performed using Affymetrix arrays (U133 Human Chip Plus 2.0) according to the manufacturer's instructions (see Supplemental Methods). Human and mouse array data were deposited in NCBI's Gene Expression Omnibus (see Supplemental Methods).

\section{Statistical analysis}

Data are expressed as mean \pm SEM or SD. Student's t-test, ANOVA, Mann-Whitney and Chi-square (3-way contingency table) were calculated to compare experimental groups. The probability curves of survival and recurrence were calculated according to Kaplan-Meier and compared by log-rank test. Differences were considered as statistically significant if $\mathrm{p}<0.05$. Analyses were conducted using R statistical package (www.r-project.org) and SPSS® software (version 14).

\section{Results}

\section{Reduced survival in patients with HCCs expressing very low levels of KLF6 mRNA}

We previously reported that KLF6 mRNA expression was decreased in advanced HCC (4); however, these findings did not indicate whether reduced KLF6 expression affected clinical outcomes. To address this issue we correlated KLF6 mRNA with clinical outcomes in a cohort of 56 patients with HCV-related HCC following tumor resection, in whom prospective data were available from among a larger cohort of 149 patients (Figure 1A, Supplemental Table 1). We selected a subgroup representing those with 'very low' expression of KLF6 (defined by mRNA levels $<10 \%$ of normal liver, $n=16$ ) and compared their clinical outcome to patients with 'low' expression of KLF6 mRNA levels (defined by mRNA levels $>10 \%$ but $<20 \%$ of normal liver, $n=40$ ). As shown in Figure 1B, very low KLF6 mRNA expression was correlated with reduced survival $(\mathrm{p}=0.04)$ during a median follow-up of 32 months. Additionally, there was a non-significant trend towards higher recurrence rates in patients with 'very low' very low KLF6 mRNA expression ( $\mathrm{p}=0.07)$ (Figure 1C).

\section{Reduced KLF6 mRNA expression in livers of KLF6 +/- mice}

Evidence that reduced KLF6 mRNA expression was associated with clinical outcomes in patients established a rationale to assess the impact of reduced KLF6 on tumor formation in mice following carcinogenic stress. Accordingly, male KLF6 +/- mice were exposed to a single dose of DEN, a well-validated murine model of chemically induced HCC (21).

Untreated KLF6 +/- mice had reduced hepatic KLF6 mRNA and protein expression to $39-65 \%$ of KLF6 +/+ mice (Supplemental Figures 1A and B). Interestingly, this expression 
level in KLF6 +/- mice was similar to KLF6 mRNA expression in HCV-infected cirrhotic liver (Figure 1A), further validating this model in assessing the impact of KLF6 loss on carcinogenic propensity in human HCC. Moreover, although KLF6 mRNA levels increased with age after DEN in both KLF6 +/+ and KLF6 +/- animals, levels in the KLF6 +/- animals remained significantly lower (Figure $2 \mathrm{~A}$ ).

\section{Increased liver size in KLF6 +/- mice after DEN}

While body weight was not affected by KLF6 heterozygosity, liver weight in the KLF6 +/mice after DEN increased relative to KLF6 +/+ mice $(\mathrm{p}<0.05)$ accounting for a significantly increased ratio of liver to body weight at 9 months $(\mathrm{p}<0.05$; Figure $2 \mathrm{~B}-\mathrm{D})$.

\section{Increased HCC development in KLF6 +/- mice after DEN}

We next assessed the impact of KLF6 allelic loss on tumor formation after DEN, measuring the number and size of tumors, as well as the total surface area of the liver occupied by tumors in the DEN-treated animals. No macroscopic tumors or histologic abnormalities were visible at 3 months (Figure 3A). At 6 months, macroscopic tumors were clearly evident in $20 \%$ of KLF6 +/- mice but none were present in KLF6 +/+ animals, whereas at 9 months tumor prevalence was $71 \%$ in KLF6 +/- mice, compared with $40 \%$ in KLF6 +/+ animals $(\mathrm{p}<0.01$; Table 1 and Figure 3A). Interestingly, the number, size, and relative surface area occupied by the tumors were significantly increased in KLF6 +/- mice, compared to ageand sex-matched wild type animals treated with DEN (Figures 3B, C and D); $(\mathrm{p}<0.05)$; in the KLF6 +/- mice, the livers were largely replaced by tumors.

\section{Increased liver injury in KLF6 +/- mice after DEN}

There was evidence of increased liver injury associated with HCC progression in KLF6 +/mice compared to wild type animals, as assessed by serum alkaline phosphatase, AST and ALT. These increased liver biochemistries were evident at 9 months $(\mathrm{p}<0.05)$, indicating that loss of a KLF6 allele may amplify the inflammatory effect of DEN (Figure 3E). As expected, the effects of KLF6 allelic loss on tumor propensity were present only in male mice. In contrast, in female KLF6 +/- mice treated with DEN there was no difference in tumor formation versus KLF6 +/+ animals even at 12 and 15 months, although overall tumor frequency was significantly lower than in male mice (data not shown).

\section{KLF6 heterozygosity generates a poor-prognosis gene-expression signature in non- malignant livers following DEN}

We hypothesized that carcinogenic signaling networks might be amplified in KLF6 +/livers following DEN. To explore this possibility in an unbiased manner, we compared transcriptomic profiling of non-tumoral and tumoral tissues between KLF6 +/- and KLF6 +/ + mice at 6 months after DEN, reasoning that changes in gene expression favoring tumorigenesis were likely to be present before HCC developed.

We previously reported a 186-gene-expression signature predictive of poor survival following surgical resection of human HCC (22). The signature was identified in cirrhotic liver, not tumor tissues, and is thought to reflect a carcinogenic "field effect" present in the diseased liver prior to the development of HCC. We hypothesized that KLF6 deficiency might promote a comparably permissive microenvironment in murine liver. Indeed, in mice treated with DEN, loss of a single KLF6 allele generated a gene signature in non-malignant liver that is highly predictive of poor-survival in human HCC, compared to wild type mice treated with DEN (FDR=0.05, Supplemental Figure 2). These findings support the notion that loss of KLF6 favors the emergence of HCC under carcinogenic stress, with molecular defects similar to the findings in human HCC. 


\section{HCCs in KLF6 +/- mice reproduce the gene signatures of highly aggressive human HCC}

Recent genomic profiling of human HCC tumor tissues has revealed distinct molecular subclasses of HCC, which discriminate tumors with different behavior and outcomes (23). With these classifications in mind, we explored whether features of any of the HCC subclasses were present in the KLF6 +/- tumors induced by DEN. Remarkably, signatures independently associated with aggressive human $\mathrm{HCC}$ behavior were over-expressed in KLF6 +/- tumors compared to wild type KLF6 tumors, whereas signatures associated with less-aggressive tumor behavior were under-expressed (Supplemental Table 2 and Supplemental Figure 2).

\section{KLF6 loss is linked to increased mdm2 expression and reduced p53 activity}

There was evidence of altered expression in KLF6 +/- tumors of transcripts related to the cell cycle, the p53 pathway and inflammation. In particular, $m d m 2$ mRNA expression was increased, reflecting dysregulation of a major pathway that controls p53 half-life and activity (24). Expression of $m d m 2$ mRNA at 6 months was increased $72 \%$ in KLF6 +/- tumor tissue compared with tumors in KLF6 +/+ mice ( $\mathrm{p}<0.01)$, and increased $40 \%$ in KLF6 +/- tumors versus surrounding tissue $(\mathrm{p}=0.05)$. We speculated that KLF6 might normally trans-repress $m d m 2$ gene expression, whereas reduced KLF6 expression would de-repress $m d m 2$, leading to increased degradation of p53 and attenuation of its tumor suppressive activity. Indeed, after DEN, $m d m 2$ mRNA was significantly increased in KLF6 +/- non-tumoral livers compared to KLF6 +/+ (Figure 4A), associated with increased expression of MDM2 protein and reduced expression of p53 (Figure 4B). The reduction of p53 was associated with downregulation p21 (both a p53 and KLF6 target gene), as well as p53-specific target genes PUMA, and cyclin G (Figure 4C-D). To directly link KLF6 to p53 regulation, primary mouse hepatocytes were transfected with a KLF6 cDNA, which led to a $~ 50 \%$ decrease in MDM2 (the $72 \mathrm{kDa}$ band, which derives from the KLF6-responsive $m d m 2$ transcript) and increased p53 protein levels (Figure 4E).

\section{Transcriptional repression of $\mathrm{mdm} 2$ by KLF6 leads to increased p53}

We examined whether the $h d m 2$ gene (human homologue of $m d m 2$ ) is a direct transcriptional target of KLF6 using chromatin immunoprecipitation (ChIP). Sequence analysis uncovered two potential KLF6 target sites ("CACCC Boxes") (2) within the $m d m 2$ P2 promoter (Figure 5A); by ChIP, KLF6 specifically interacted with this region upstream of exon 2. In contrast, no interaction was documented within the P1 promoter region.

To confirm that a reciprocal relationship exists between KLF6 and MDM2, we transfected a fixed amount of wild type p53 into a p53 null hepatoma cell line (Hep3B), while modulating KLF6 expression: KLF6 over-expression led to decreased $m d m 2$ promoter activity, whereas KLF6 knockdown had the opposite effect ( $\mathrm{p}<0.05$, Figure 5B). Moreover, KLF6 effect on $m d m 2$ expression was specifically correlated with the interaction of KLF6 with P2 promoter of $m d m 2$ (Figure 5C). To examine whether KLF6-mediated repression of $m d m 2$ affects p53 activity, we co-transfected KLF6 cDNA into three HCC cell lines with a p53-responsive promoter derived from the cyclin G gene (20). As predicted, in HepG2 and Huh7 cells, which have partially functional p53 (25), there was marked transactivation of cyclin G luciferase in the presence of KLF6. In contrast, Hep3B cells, which are p53 null, lacked transcriptional activity in response to KLF6 transfection (Figure 5D).

Finally, since loss of KLF6 occurs in progressive hepatic carcinogenesis, we predicted that there should be a reciprocal relationship between progressively decreased KLF6 expression and rising levels of $m d m 2$ mRNA expression in human HCC. To test this possibility, we examined microarray data from a well-characterized cohort of HCV patients (15). As predicted, there was a progressive increase in $m d m 2 \mathrm{mRNA}$ expression during the 
progression from normal liver to dysplastic nodules, associated with declining KLF6 mRNA expression (Figure 6A). Interestingly, $m d m 2$ mRNA levels did not correlate with survival (data not shown), indicating that the impact of KLF6 loss on survival is not mediated solely by its effects on mdm 2 expression. To further evaluate this reciprocal correlation between KLF6 and MDM2, we co-transfected Hep3B with a fixed amount of p53 and KLF6: the over-expression of KLF6 within a physiological range (less than 2-fold) was able to reduce $m d m 2$ mRNA expression levels by $\sim 50 \%$ (Figure $6 \mathrm{~B}$ ). While this finding does not establish that KLF6 loss is the only determinant of rising $m d m 2$ mRNA expression in human hepatocarcinogenesis, it nonetheless indicates that loss contributes to enhanced $m d m 2$ expression and loss of p53 function.

\section{Discussion}

We have demonstrated that KLF6 deficiency promotes DEN-induced HCC in mice, which closely models gene signatures in both tumors and surrounding tissues that are associated with poor outcomes in human HCC. These findings reinforce the emerging importance of KLF6 as a tumor suppressor in HCC, and suggest that KLF6 loss may contribute to a ffield effect' in promoting a carcinogenic milieu during chronic liver injury. The association of KLF6 deficiency with more aggressive human HCC subclasses (S1 and S2)(23, 26) implicates this gene in the stepwise malignant transformation and dedifferentiation associated with hepatic tumorigenesis. Moreover, these subclasses are associated with impaired p53 function (Supplemental Figure 3), consistent with the link we have uncovered between KLF6 and p53.

The findings also indicate that KLF6 functions as a tumor suppressor in part through its repression of MDM2, which negatively regulates p53 activity and induces p53 degradation. The data further suggest a potential prognostic link between reduced KLF6 expression in HCC and poor survival, underscoring its functional importance in this neoplasm. Progressive HCC is associated with reduced KLF6 mRNA expression in a large majority of HCCs associated with HCV infection (4). We modeled this condition in mice by analyzing the response of KLF6 +/- animals to the carcinogen DEN, confirming a markedly increased tumor burden, and uncovering $m d m 2$ as a novel target gene of KLF6 using microarray and ChIP. Importantly, loss of KLF6 leads to reduced trans-repression of $m d m 2$, thereby promoting loss of $\mathrm{p} 53$ through its accelerated degradation. The findings provide a novel pathway by which MDM2 activity is enhanced in human cancer, adding to other mechanisms that increase MDM2 expression, with or without gene amplification (13). Moreover, since KLF6 down-regulation is recognized in a growing number of human cancers, this pathway may contribute to enhanced MDM2 activity in a range of tumors. At least one mechanism by which down-regulation of KLF6 occurs is through MYC amplification (27), however other determinants are also likely. In particular, KLF6 downregulation through promoter methylation occurs in esophageal cancer cells (28).

Reciprocal expression of $K L F 6$ and $m d m 2$ mRNAs was also demonstrated in human HCVrelated $\mathrm{HCC}$, in which there was a progressive increase of $m d m 2 \mathrm{mRNA}$ that paralleled progressive diminution of KLF6 mRNA. Interestingly, this correlation was strongest in lowgrade dysplastic nodules, which are critical pre-malignant stages from which the tumor phenotype emerges (29). Similarly, in mice the correlation was greatest at 6 months after DEN, before tumor formation was maximal. These findings are clinically important, since $\mathrm{HCV}$-associated tumors arise in a step-wise fashion, and almost exclusively develop once advanced fibrosis or cirrhosis is present (30). Additionally, a growing body of evidence implicates chronic inflammation in the pathogenesis of HCC (31-33). In that context, it is noteworthy that KLF6 haploinsufficiency led to increased elevations in enzymes indicative 
of liver injury, raising the possibility that part of the tumor-promoting effect of KLF6 depletion involves amplified inflammatory signaling.

The maximal impact of $m d m 2$ de-repression through KLF6 deficiency would be expected only if p53 is not mutated. Indeed, inactivating mutations of p53 in HCC are relatively uncommon, and typically occur at late stages of the disease (34-35). Moreover, the likelihood of p53 abnormalities varies considerably among different HCC etiologies (36-37) and has not been studied extensively in HCV (38), which is the most common etiology of the disease in the Western world and the sole cause among the tumors analyzed in our study. Other mechanisms of $\mathrm{HCV}$ pathogenesis have also been linked to p53 (38), and it remains possible that KLF6 might also interact with HCV directly. Additionally, since all the HCCs in this study were derived from patients with HCV infection, a link between KLF6 and MDM2 cannot be assumed for other etiologies, in particular hepatitis B and fatty liver, even though the relationship was also documented in DEN-induced neoplasia.

In summary, our findings uncover a new pathway of tumor suppression in HCC, in which loss of KLF6 activity leads to increased MDM2 and accelerated degradation of p53. The convergence of these two tumor suppressor pathways underscores the highly interdependent molecular abnormalities that characterize this neoplasm.

\section{Supplementary Material}

Refer to Web version on PubMed Central for supplementary material.

\section{Acknowledgments}

We wish to acknowledge Rachel Schwartz and Romina Bromberg for assistance with the human HCC sample analysis.

GRANT Support: The work was supported by the National Institutes of Health (RO1DK37340 and RO1DK56621 to S.L.F, R01DK076986 to J.M.L.). R.H. was funded by the Graduate School for Drug Exploration (GUIDE), and the Stichting Nicolaas Muleriusfonds, Groningen, the Netherlands. A.V. is a recipient of a Sheila Sherlock fellowship from the European Association for the Study of the Liver. D.V. is a recipient of a research fellowship from the Swiss National Fund. G.N. is a recipient of the Howard Hughes Medical Institute Physician-Scientist Early Career Award.

\section{References}

1. Vinciguerra M, Foti M. PTEN at the crossroad of metabolic diseases and cancer in the liver. Ann Hepatol. 2008 Jul-Sep.7:192-199. [PubMed: 18772845]

2. Narla G, Heath KE, Reeves HL, Li D, Giono LE, Kimmelman AC, Glucksman MJ, et al. KLF6, a candidate tumor suppressor gene mutated in prostate cancer. Science. 2001; 294:2563-2566. [PubMed: 11752579]

3. DiFeo A, Narla G, Hirshfeld J, Camacho-Vanegas O, Narla J, Rose SL, Kalir T, et al. Roles of KLF6 and KLF6-SV1 in ovarian cancer progression and intraperitoneal dissemination. Clin Cancer Res. 2006; 12:3730-3739. [PubMed: 16778100]

4. Kremer-Tal S, Narla G, Chen Y, Hod E, Difeo A, Yea S, Lee JS, et al. Downregulation of KLF6 is an early event in hepatocarcinogenesis, and stimulates proliferation while reducing differentiation. $\mathrm{J}$ Hepatol. 2007; 46:645-654. [PubMed: 17196295]

5. Camacho-Vanegas O, Narla G, Teixeira MS, DiFeo A, Misra A, Friedman S, Feuerstein BG, et al. Functional inactivation of the KLF6 tumor suppressor gene by loss of heterozygosity and increased alternative splicing in glioblastoma. Int J Cancer. 2007 in press.

6. Bieker JJ. Kruppel-like factors: three fingers in many pies. J Biol Chem. 2001; 276:34355-34358. [PubMed: 11443140] 
7. Benzeno S, Narla G, Allina J, Cheng GZ, Reeves HL, Banck MS, Odin JA, et al. Cyclin-dependent kinase inhibition by the KLF6 tumor suppressor protein through interaction with cyclin D1. Cancer Res. 2004; 64:3885-3891. [PubMed: 15172998]

8. Slavin DA, Koritschoner NP, Prieto CC, Lopez-Diaz FJ, Chatton B, Bocco JL. A new role for the Kruppel-like transcription factor KLF6 as an inhibitor of c-Jun proto-oncoprotein function. Oncogene. 2004; 23:8196-8205. [PubMed: 15378003]

9. Katiyar S, Dash BC, Thakur V, Guptan RC, Sarin SK, Das BC. P53 tumor suppressor gene mutations in hepatocellular carcinoma patients in India. Cancer. 2000; 88:1565-1573. [PubMed: 10738214]

10. Shmueli A, Oren M. Mdm2: p53's lifesaver? Mol Cell. 2007; 25:794-796. [PubMed: 17386256]

11. Juven T, Barak Y, Zauberman A, George DL, Oren M. Wild type p53 can mediate sequencespecific transactivation of an internal promoter within the mdm2 gene. Oncogene. 1993; 8:34113416. [PubMed: 8247544]

12. Lahav G. Oscillations by the p53-Mdm2 feedback loop. Adv Exp Med Biol. 2008; 641:28-38. [PubMed: 18783169]

13. Michael D, Oren M. The p53 and Mdm2 families in cancer. Curr Opin Genet Dev. 2002; 12:5359. [PubMed: 11790555]

14. Llovet JM, Chen Y, Wurmbach E, Roayaie S, Fiel MI, Schwartz M, Thung SN, et al. A molecular signature to discriminate dysplastic nodules from early hepatocellular carcinoma in HCV cirrhosis. Gastroenterology. 2006; 131:1758-1767. [PubMed: 17087938]

15. Chiang DY, Villanueva A, Hoshida Y, Peix J, Newell P, Minguez B, LeBlanc AC, et al. Focal gains of VEGFA and molecular classification of hepatocellular carcinoma. Cancer Res. 2008; 68:6779-6788. [PubMed: 18701503]

16. Rimkus C, Martini M, Friederichs J, Rosenberg R, Doll D, Siewert JR, Holzmann B, et al. Prognostic significance of downregulated expression of the candidate tumour suppressor gene SASH1 in colon cancer. Br J Cancer. 2006; 95:1419-1423. [PubMed: 17088907]

17. Narla G, Kremer-Tal S, Matsumoto N, Zhao X, Yao S, Kelley K, Tarocchi M, et al. In vivo regulation of p21 by the Kruppel-like factor 6 tumor-suppressor gene in mouse liver and human hepatocellular carcinoma. Oncogene. 2007; 26:4428-4434. [PubMed: 17297474]

18. Narla G, DiFeo A, Yao S, Banno A, Hod E, Reeves HL, Qiao RF, et al. Targeted inhibition of the KLF6 splice variant, KLF6 SV1, suppresses prostate cancer cell growth and spread. Cancer Res. 2005; 65:5761-5768. [PubMed: 15994951]

19. Zauberman A, Flusberg D, Haupt Y, Barak Y, Oren M. A functional p53-responsive intronic promoter is contained within the human mdm2 gene. Nucleic Acids Res. 1995; 23:2584-2592. [PubMed: 7651818]

20. Zauberman A, Lupo A, Oren M. Identification of p53 target genes through immune selection of genomic DNA: the cyclin G gene contains two distinct p53 binding sites. Oncogene. 1995; 10:2361-2366. [PubMed: 7784084]

21. Naugler WE, Sakurai T, Kim S, Maeda S, Kim K, Elsharkawy AM, Karin M. Gender disparity in liver cancer due to sex differences in MyD88-dependent IL-6 production. Science. 2007; 317:121124. [PubMed: 17615358]

22. Hoshida Y, Villanueva A, Kobayashi M, Peix J, Chiang DY, Camargo A, Gupta S, et al. Gene expression in fixed tissues and outcome in hepatocellular carcinoma. N Engl J Med. 2008; 359:1995-2004. [PubMed: 18923165]

23. Hoshida Y, Toffanin S, Lachenmayer A, Villanueva A, Minguez B, Llovet JM. Molecular classification and novel targets in hepatocellular carcinoma: recent advancements. Semin Liver Dis. 2010; 30:35-51. [PubMed: 20175032]

24. Haupt Y, Maya R, Kazaz A, Oren M. Mdm2 promotes the rapid degradation of p53. Nature. 1997; 387:296-299. [PubMed: 9153395]

25. Hsieh JL, Wu CL, Lee CH, Shiau AL. Hepatitis B virus $\times$ protein sensitizes hepatocellular carcinoma cells to cytolysis induced by E1B-deleted adenovirus through the disruption of p53 function. Clin Cancer Res. 2003; 9:338-345. [PubMed: 12538486] 
26. Hoshida Y, Nijman SM, Kobayashi M, Chan JA, Brunet JP, Chiang DY, Villanueva A, et al. Integrative transcriptome analysis reveals common molecular subclasses of human hepatocellular carcinoma. Cancer Res. 2009; 69:7385-7392. [PubMed: 19723656]

27. Sole X, Hernandez P, Lopez de Heredia M, Armengol L, Rodriguez-Santiago B, Gomez L, Maxwell CA, et al. Genetic and genomic analysis modeling of germline c-MYC overexpression and cancer susceptibility. BMC Genomics. 2008; 9:12. [PubMed: 18190704]

28. Yamashita K, Upadhyay S, Osada M, Hoque MO, Xiao Y, Mori M, Sato F, et al. Pharmacologic unmasking of epigenetically silenced tumor suppressor genes in esophageal squamous cell carcinoma. Cancer Cell. 2002; 2:485-495. [PubMed: 12498717]

29. Kojiro M. Premalignant lesions of hepatocellular carcinoma: pathologic viewpoint. J Hepatobiliary Pancreat Surg. 2000; 7:535-541. [PubMed: 11180884]

30. Yoshida H, Shiratori Y, Moriyama M, Arakawa Y, Ide T, Sata M, Inoue O, et al. Interferon therapy reduces the risk for hepatocellular carcinoma: national surveillance program of cirrhotic and noncirrhotic patients with chronic hepatitis C in Japan. IHIT Study Group. Inhibition of Hepatocarcinogenesis by Interferon Therapy. Ann Intern Med. 1999; 131:174-181. [PubMed: 10428733]

31. Karin M. NF-kappaB and cancer: mechanisms and targets. Mol Carcinog. 2006; 45:355-361. [PubMed: 16673382]

32. Luedde T, Trautwein C. A molecular link between inflammation and fibrogenesis: the bacterial microflora influences hepatic fibrosis via toll-like receptor 4-dependent modification of transforming growth factor-beta signaling in hepatic stellate cells. Hepatology. 2008; 47:1089_ 1091. [PubMed: 18306215]

33. Pikarsky E, Porat RM, Stein I, Abramovitch R, Amit S, Kasem S, Gutkovich-Pyest E, et al. NFkappaB functions as a tumour promoter in inflammation-associated cancer. Nature. 2004; 431:461-466. [PubMed: 15329734]

34. Hosono S, Chou MJ, Lee CS, Shih C. Infrequent mutation of p53 gene in hepatitis B virus positive primary hepatocellular carcinomas. Oncogene. 1993; 8:491-496. [PubMed: 8093978]

35. Ng IO, Chung LP, Tsang SW, Lam CL, Lai EC, Fan ST, Ng M. p53 gene mutation spectrum in hepatocellular carcinomas in Hong Kong Chinese. Oncogene. 1994; 9:985-990. [PubMed: 8108145]

36. Buendia MA. Genetics of hepatocellular carcinoma. Semin Cancer Biol. 2000; 10:185-200. [PubMed: 10936068]

37. Suriawinata A, Xu R. An update on the molecular genetics of hepatocellular carcinoma. Semin Liver Dis. 2004; 24:77-88. [PubMed: 15085488]

38. McGivern DR, Lemon SM. Tumor suppressors, chromosomal instability, and hepatitis C virusassociated liver cancer. Annu Rev Pathol. 2009; 4:399-415. [PubMed: 18928409]

\section{List of Abbreviations}

$\begin{array}{ll}\text { ALT } & \text { alanine transaminase } \\ \text { AST } & \text { aspartate transaminase } \\ \text { ChIP } & \text { chromatin immunoprecipitation } \\ \text { DEN } & \text { diethyl nitrosamine } \\ \text { HCC } & \text { hepatocellular carcinoma } \\ \text { HCV } & \text { hepatitis C virus } \\ \text { LOH } & \text { loss of heterozygosity }\end{array}$



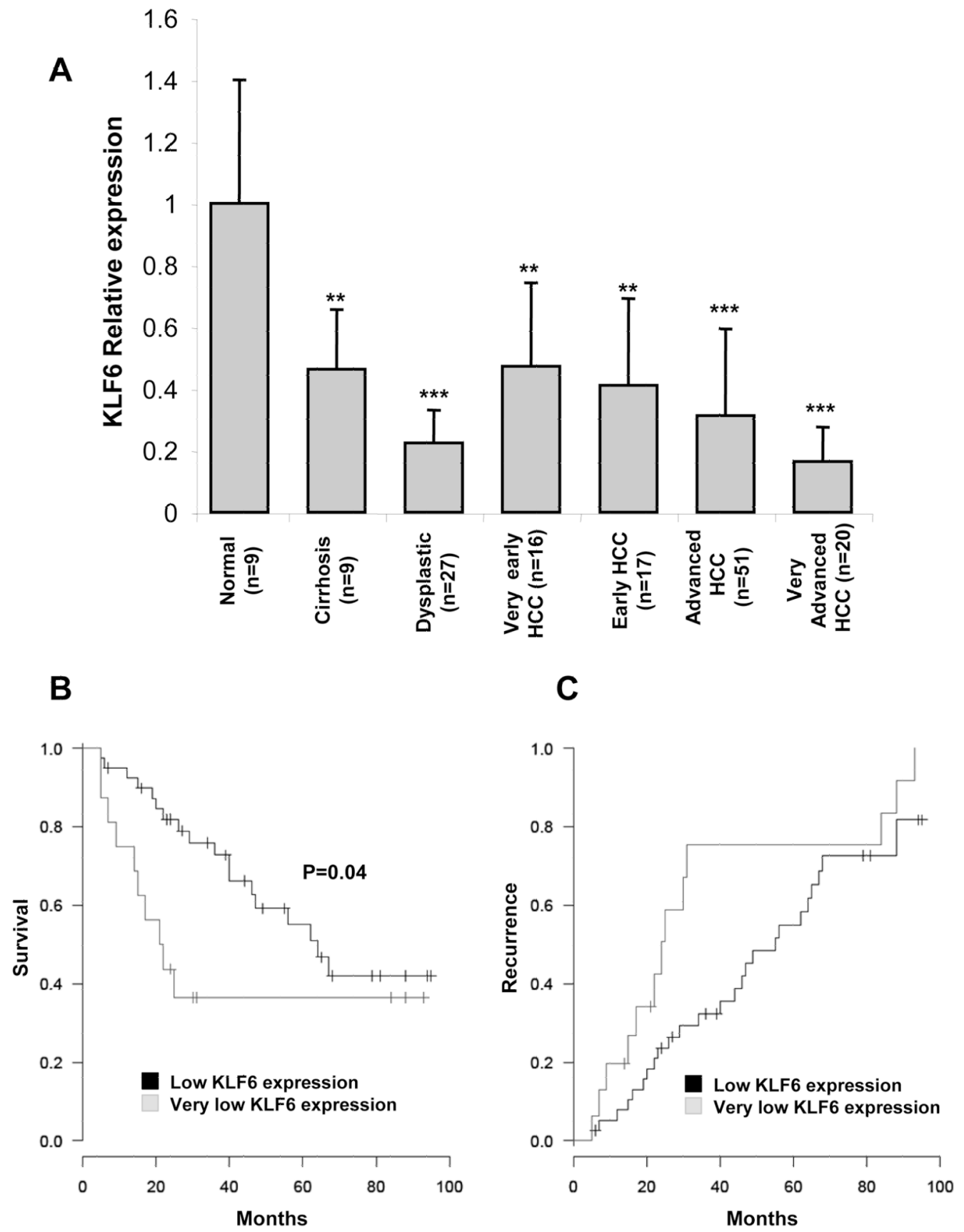

Figure 1. Correlation between KLF6 expression level and stage and prognosis in HCV patient (A) KLF6 mRNA expression was quantified in 149 liver samples from HCV-infected patients representing the histologic spectrum of lesions associated with progressive $\mathrm{HCV}$ liver disease. RT-qPCR was performed using primers targeting KLF6 full length form, and data are expressed as relative expression compared to "normal" liver, normalized to GAPDH mRNA \pm SD $(* * \mathrm{p}<0.01, * * * \mathrm{p}<0.001)$.

(B) KLF6 expression was correlated with progression in resected HCCs in 56 patients over 8 year period: patients with 'very low expression' $(n=16)$ had a reduced overall survival compared to patients with 'low expression' $(n=40)(p<0.05)$. 
(C) There was a trend towards higher recurrence rates in patients with very low expression levels of KLF6 ( $\mathrm{p}=0.07)$. 

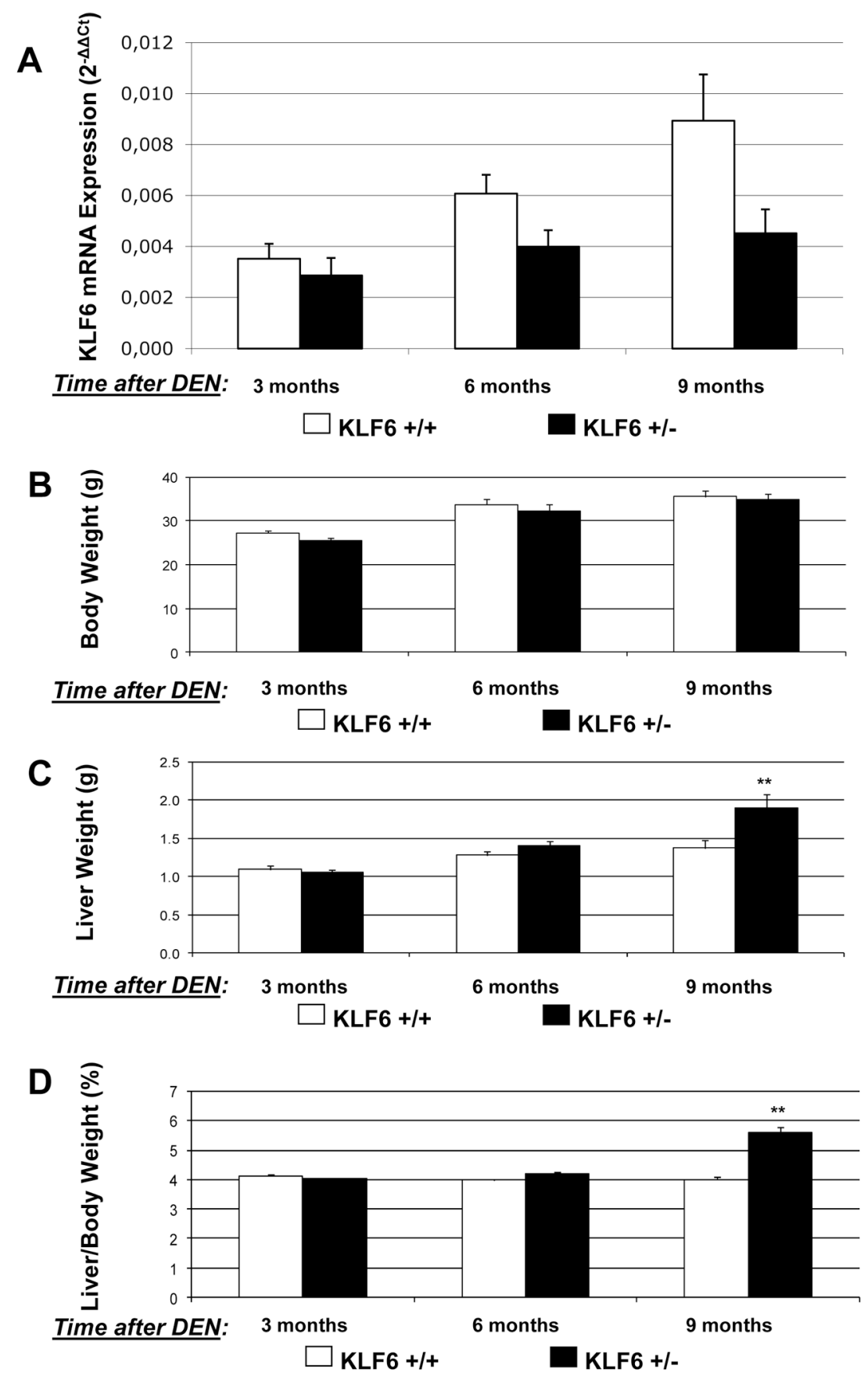

Figure 2. KLF6 expression and liver/body weight changes in mice after DEN treatment (A) Analysis of KLF6 mRNA levels over a period of 9 months in animals following a single injection of $5 \mu \mathrm{g} / \mathrm{g}$ DEN i.p. at 2 weeks of age. mRNA data are depicted as relative expression $( \pm$ SEM), normalized to GAPDH mRNA ( $\mathrm{n} \geq 8$ for each group).

(B) Total body weight of the animals was measured at the time of sacrifice; values are expressed in grams as average \pm SEM (numbers listed in Table 1).

(C) Total liver weight at time of sacrifice, expressed in grams as average $\pm \operatorname{SEM}(* * \mathrm{p}<0.01)$.

(D) Ratio between total liver weight and total body weight is expressed in \% as average $\pm \operatorname{SEM}(* * \mathrm{p}<0.01)$. 
A
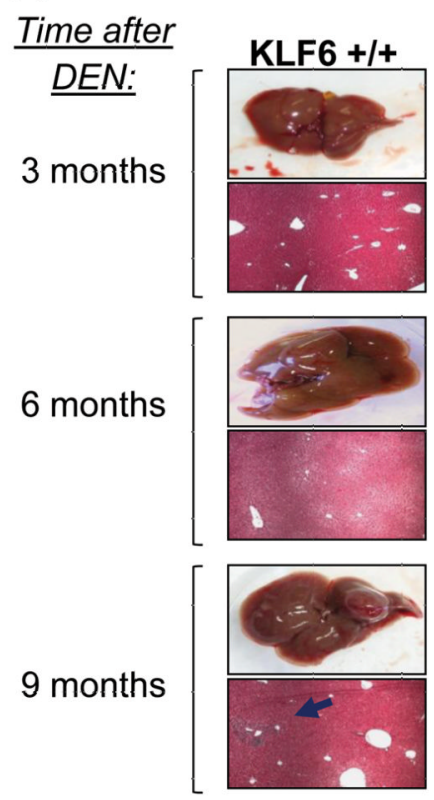

\section{E}
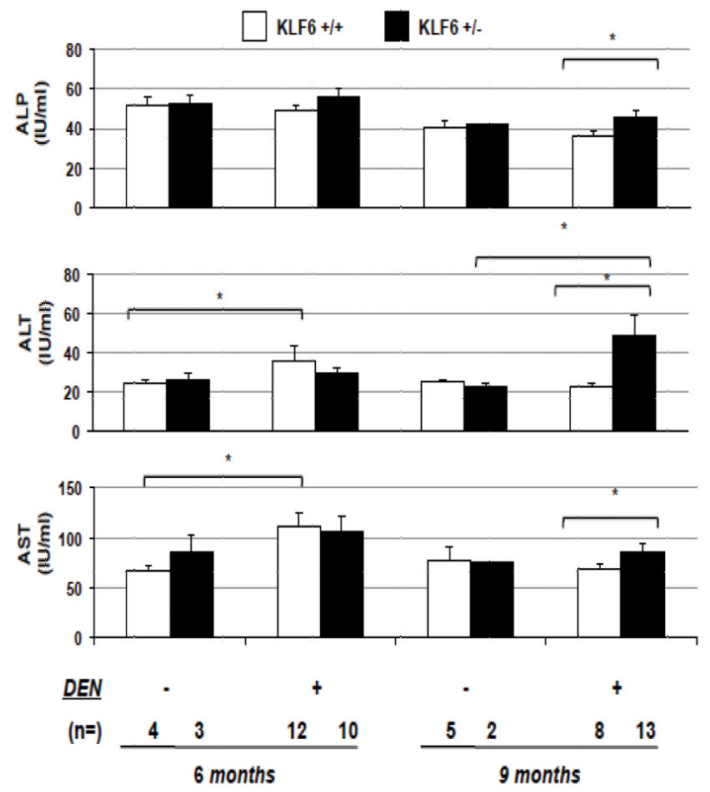

B

KLF6+/-
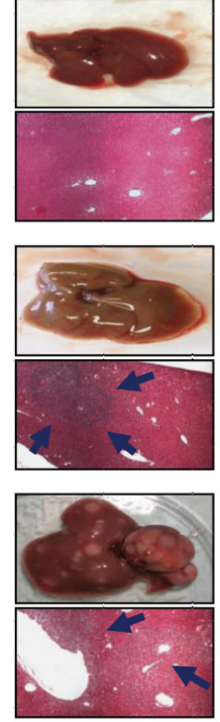

C $_{1.8}$

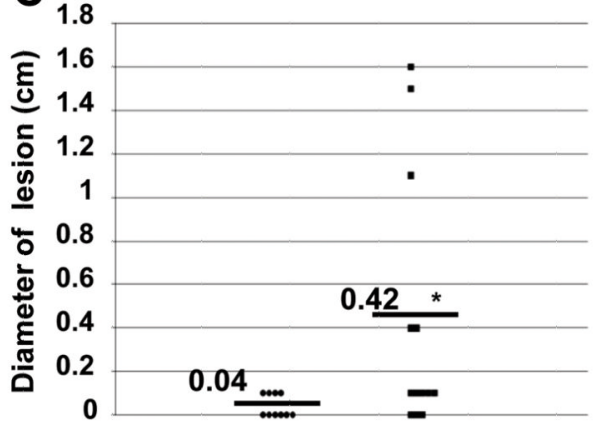

KLF6 +/+ KLF6+/-

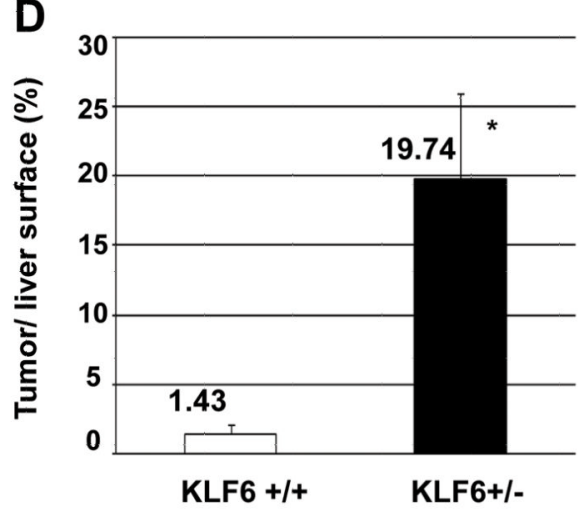

Figure 3. Increased susceptibility of KLF6 +/- mice to DEN-induced HCC

(A) Representative images of livers at 3, 6, and 9 months after DEN treatment. Microscopic tumors were identified following H\&E staining (indicated with arrows; $20 \times$ magnification). (B) Number of macroscopic tumors $(\geq 0.1 \mathrm{~cm})$ identified in 9-month-old animals at the time of sacrifice. Data are expressed as average of tumor numbers for each group $(* \mathrm{p}<0.05)$.

(C) The diameter $(\mathrm{cm})$ of the macroscopic tumors were measured with a caliper $(* \mathrm{p}<0.05)$.

(D) Tumor surface was estimated in the left lobe of each liver by direct measurement, and the surface ratio between the tumor and the lobe was quantified with an image analyzer (BioQuant Software) (expressed as average \pm SEM; * ${ }^{*}<0.05$ ). 
(E) Biochemical analysis was performed on serum from mice harvested at the time of sacrifice. There were significant differences between KLF6 +/+ and KLF6 +/- mice at 9 months in alkaline phosphatase (ALP), alanine aminotransaminase (ALT) and aspartate aminotransaminase (AST) levels $(* \mathrm{p}<0.05)$. 


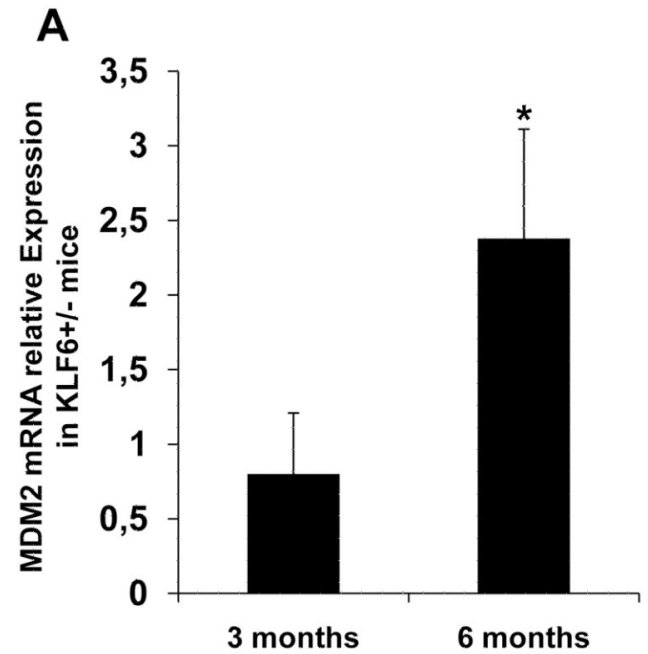

B 3 months 6 months

$$
+/++/-+1++/-
$$

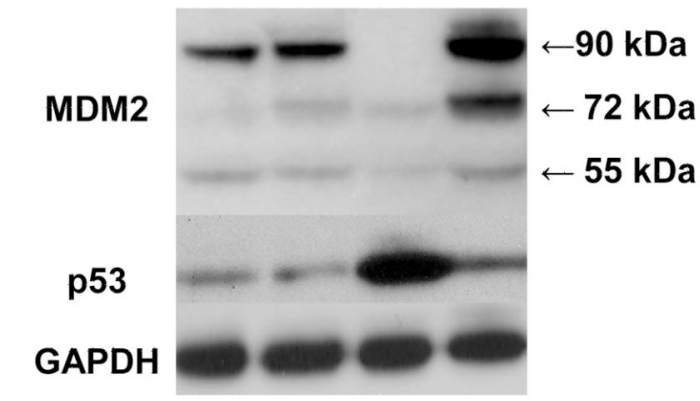

C 3 months 6 months

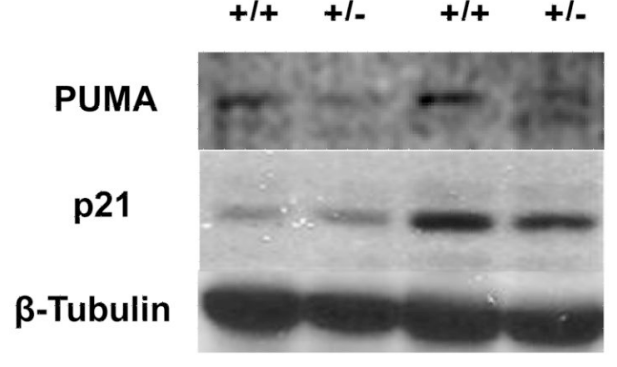
relative expression \pm SEM $(* \mathrm{p}<0.05)$. loading control.
D

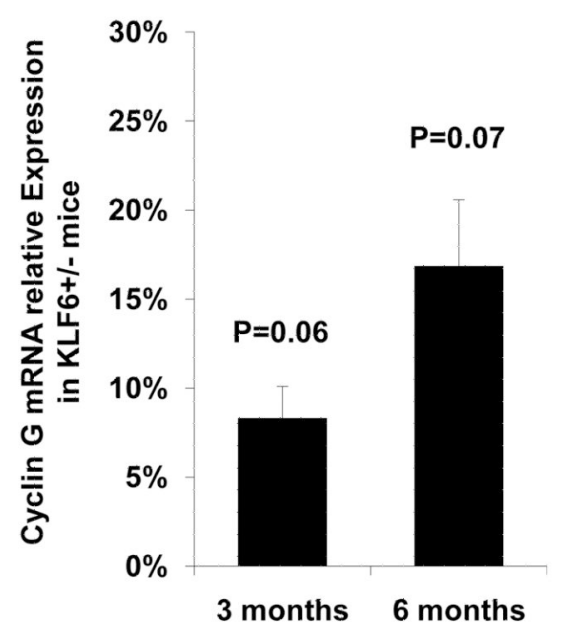

$\mathbf{E}$
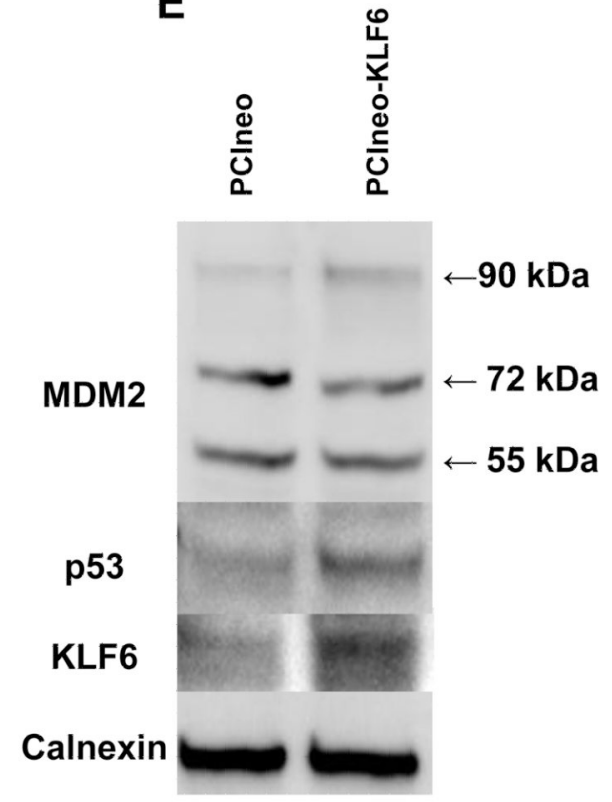

Figure 4. KLF6 expression is correlated with $\mathrm{mdm} 2$ and $\mathrm{p53}$ levels in liver

(A) mRNA from non-tumoral liver was isolated and $m d m 2$ expression was quantified by RT-qPCR and normalized to GAPDH: average expression in the KLF6 +/- animals was normalized to control groups matched for age ( $\mathrm{n}=6$ for each group). Values are expressed as

(B) Representative expression of MDM2 and p53 protein levels assessed by Western Blots in non-tumoral mouse liver at 3 and 6 months after DEN injection. GAPDH was used a

(C) Representative expression of PUMA and p21 protein levels assessed by Western Blots in non-tumoral mouse liver at 3 and 6 months after DEN injection. $\beta$-Tubulin was used a loading control. 
(D) mRNA from non-tumoral liver was isolated and cyclin G mRNA expression was quantified by RT-qPCR and normalized to cyclophilin mRNA: average expression in the KLF6 +/- animals was normalized to KLF6 +/+ mouse after DEN, matched for age ( $\mathrm{n}=8$ for each group). Values are expressed expression relative to age-matched KLF6 +/+ mice after DEN (set at $100 \%), \pm$ SEM.

(E) Primary hepatocytes from normal mouse liver were transfected 12 hours after isolation with pBabe- or pBabe-hKLF6, and harvested 24 hours later in protein lysis buffer.

Expression of the $72 \mathrm{kDa}$ isoform of MDM2, whose transcript is generated from the KLF6responsive intronic promoter, is reduced $\sim 50 \%$ relative to calnexin. In the same cells, p53 expression is markedly increased. This experiment was repeated in 3 separate isolates with similar results. 
A
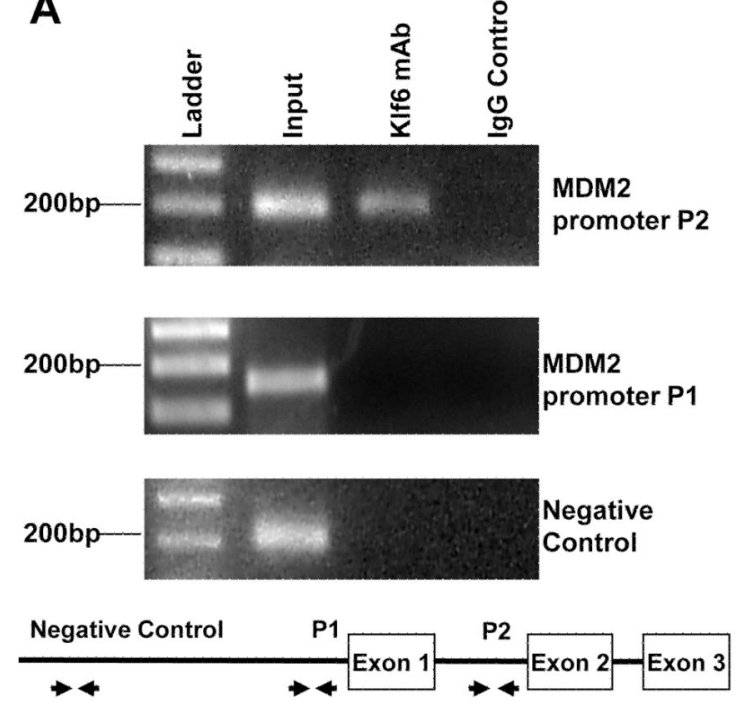

C

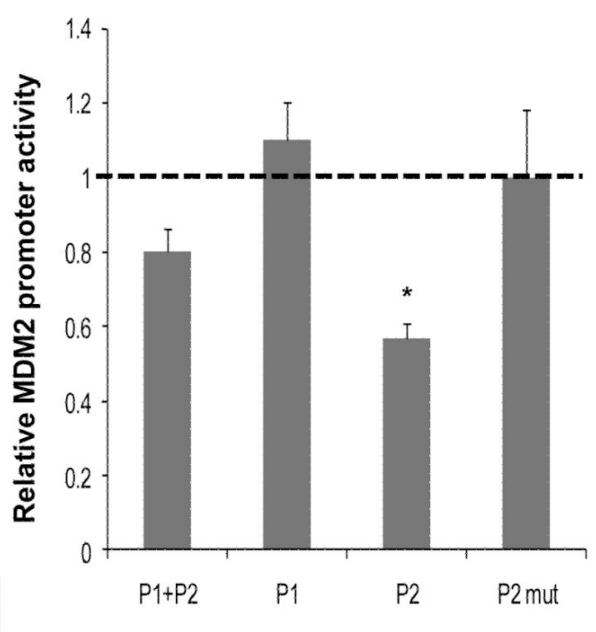

B

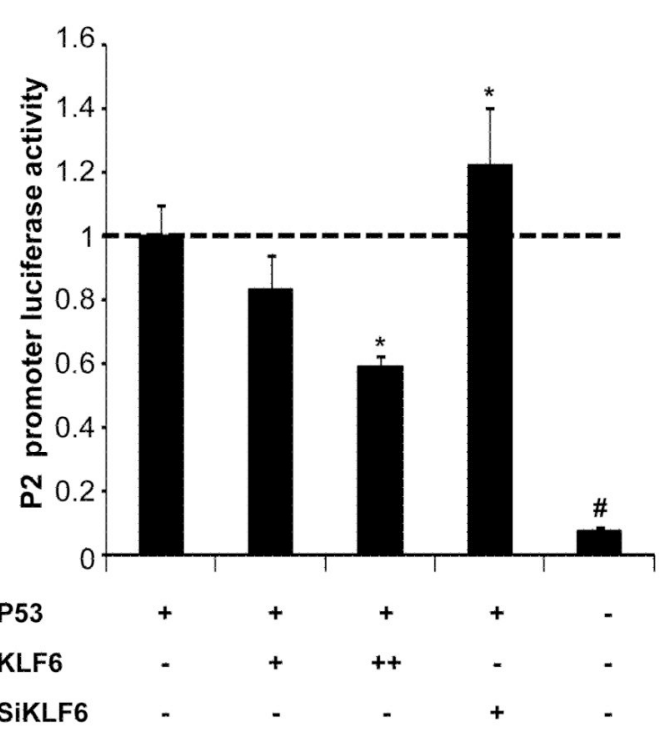

D

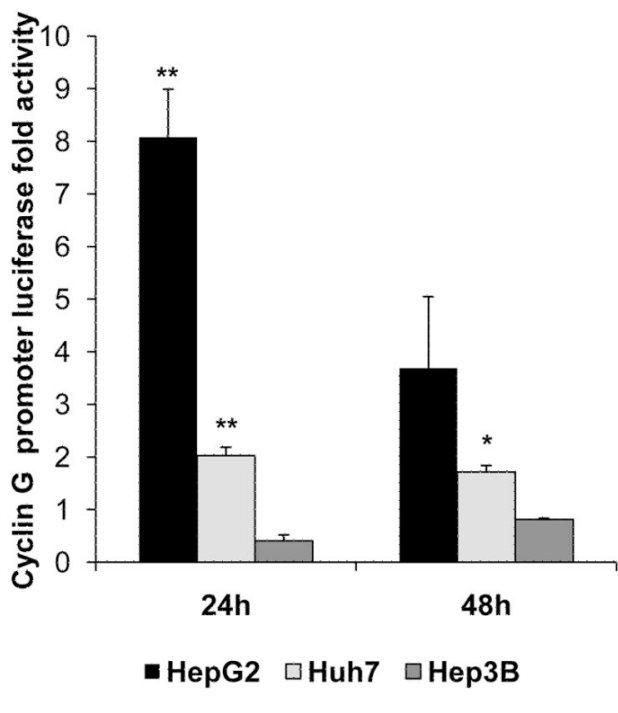

Figure 5. KLF6 directly trans-represses the mdm2 promoter

(A) Chromatin from HepG2 cell extracts was co-immunoprecipitated with anti-KLF6 antibody or IgG control antibody. Precipitated DNA was amplified using primers targeting two different regions of the human $m d m 2$ promoter: the intronic $\mathrm{P} 2$ promoter region and the upstream promoter region $\mathrm{P} 1$. The whole chromatin (input) was used as positive control. (B) Hep3B cells (p53 null) were co-transfected with a p53 expression cDNA, mdm2-P2 luciferase reporter (\#basal luciferase activity) and renilla luciferase vector (as internal control) along with either KLF6 expression cDNA (pCIneo-KLF6) or siRNA targeting full length KLF6 (pSuper-SiKLF6); representative data from 3 independent experiments is shown, each performed in triplicate $(* \mathrm{p}<0.05)$.

(C) Hep3B cells were co-transfected with p53, KLF6, renilla luciferase vector (as internal control) and luciferase reporters under the regulation of four different $m d m 2$ promoter 
regions: the whole promoter $(\mathrm{P} 1+\mathrm{P} 2)$, the $\mathrm{P} 1$ or $\mathrm{P} 2$ promoters, or $\mathrm{P} 2-\mathrm{mut}$; representative data from 3 independent experiments, each performed in triplicate $(* \mathrm{p}<0.05)$.

(D) HepG2, Huh7 and Hep3B were co-transfected with pCIneo or pCIneo-KLF6, the p53responsive cyclin G-luciferase reporter and renilla luciferase vector (as internal control); representative data from 3 independent experiments, each performed in triplicate, is shown. Whereas experiments in HepG2 and Huh7 demonstrate activation of the p53 responsive reporter by KLF6, activation of the cyclin G-luciferase was absent in Hep3B, which lack $\mathrm{p} 53(* \mathrm{p}<0.05, * * \mathrm{p}<0.01)$. 

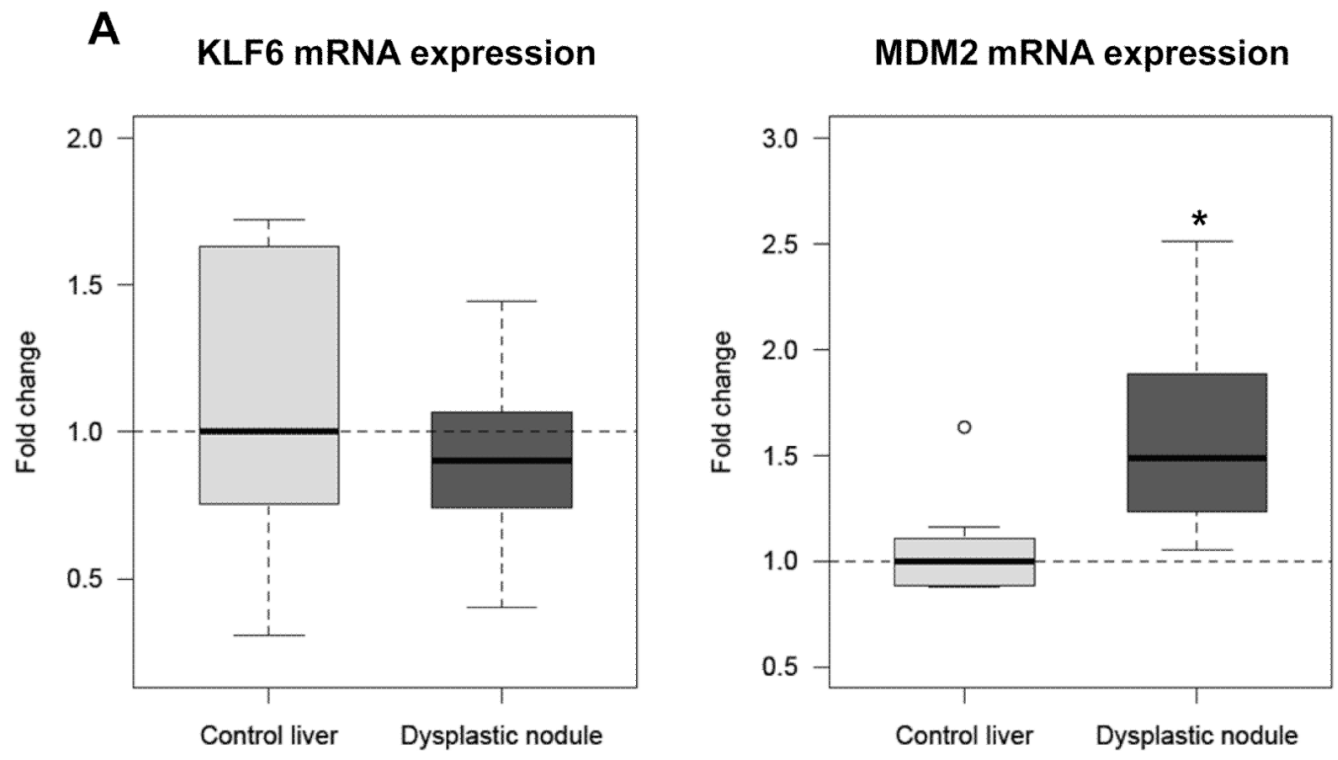

B

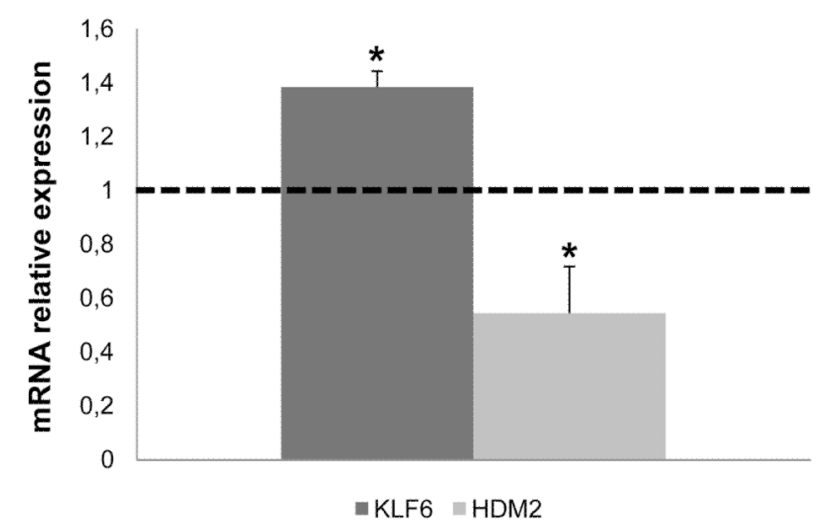

Figure 6. Reciprocal expression of KLF6 and mdm2 mRNAs in human hepatic cells (A) Human sample analysis was performed by Affymetrix U133 Plus 2.0 microarray on normal liver $(n=10)$, low grade dysplastic nodules (LGDN; $n=10)$ and high grade dysplastic nodules (HGDN; $\mathrm{n}=8$ ). Data shows fold change in KLF6 and $m d m 2$ mRNA expression during the progression to HCC; statistical difference were analyzed using the Mann-Whitney test $(* \mathrm{p}<0.05)$.

(B) Hep3B cells were co-transfected with a p53 expression cDNA and KLF6 expression cDNA (pCIneo-KLF6) or empty vector (pCIneo): KLF6 and mdm2 mRNA levels were quantified by RT-qPCR and normalized to GAPDH. Data are expressed as average \pm SEM and represent 4 independent experiments $(* \mathrm{p}<0.05)$. 


\section{Table 1}

\section{Prevalence of tumors in DEN-treated mice}

Macroscopic tumors $(\geq 0.1 \mathrm{~cm})$ were enumerated in the livers; 4 sections of the left lobe were analyzed after H\&E staining at $20 \times$ magnification field to count microscopic lesions. The prevalence of tumors was compared by genotype with a modified Chi-square test ( 3 -way contingency table; ***p<0.001).

\begin{tabular}{ccccc}
\hline & \multicolumn{2}{c}{ KLF6+/+ $(\boldsymbol{n}=39)$} & \multicolumn{2}{c}{ KLF6+/- $(\boldsymbol{n}=39)$} \\
Prevalence of lesions: & Macroscopic & Microscopic & $* * *$ Macroscopic & $* * *$ Microscopic \\
\hline 3 months & $0 \%(0 / 17)$ & $0 \%(0 / 17)$ & $0 \%(0 / 15)$ & $0 \%(0 / 15)$ \\
\hline 6 months & $0 \%(0 / 12)$ & $25 \%(3 / 12)$ & $20 \%(2 / 10)$ & $60 \%(6 / 10)$ \\
\hline 9 months & $40 \%(4 / 10)$ & $50 \%(5 / 10)$ & $71 \%(10 / 14)$ & $93 \%(13 / 14)$ \\
\hline
\end{tabular}

